Rev. Elet. em Gestão, Educação e Tecnologia Ambiental (e-ISSN: 2236-1170)

\title{
COMPARAÇÃO ENTRE A DELIMITAÇÃO MANUAL E AUTOMÁTICA DA BACIA DO ARROIO CORUPÁ, RS, BRASIL
}

\author{
Laura Camila de Godoy Goergen, Mateus Sabadi Schuh, Rudiney Soares Pereira \\ ${ }^{1}$ lauragoergen@yahoo.com.br \\ ${ }^{2}$ mateuschuh@gmail.com \\ ${ }^{3}$ rudiney.s.pereira@gmail.com
}

http://dx.doi.org/10.5902/223611707277

\section{RESUMO}

Diversos impactos ambientais como desmatamento, erosão, poluição da água e a ocupação urbana desordenada estão relacionados à falta de planejamento no uso do solo em bacias hidrográficas. Para um bom planejamento e gerenciamento dos recursos hídricos é fundamental a delimitação adequada de bacias hidrográficas. Nesse sentido, este trabalho teve como objetivo analisar uma metodologia de delimitação automática de bacias hidrográficas na bacia do Arroio Corupá, Rio Grande do Sul/RS, Brasil, utilizando dados do projeto Shuttle Radar Topographic Mission (SRTM) e comparar com os valores de área obtidos na digitalização manual com base em cartas topográficas, com escala de 1:50 000. Para isso, os dados foram integrados e processados no software ArcGIS 9.2 (ESRI, 2006). A metodologia utilizada nesse processo subdividiu-se em seis etapas, sendo: preenchimento de depressões ("fill sinks"), direção de fluxo ("flow direction"), fluxo acumulado ("flow accumulation"), extração de drenagens ("conditional -> con"), ligação de cruzamentos de fluxo ("stream link") e delimitação de bacias ("watershed"). A área da bacia delimitada a partir das curvas de nível da carta topográfica foi de 29.162,4 ha e para a delimitação automática encontrou-se área total de $28.472,2$ ha, o que representa uma diferença de $2,4 \%$ da área total, 690,2 ha. Os resultados apresentam valores muito próximos confirmando ser vantajosa a automatização da delimitação de bacias hidrográficas por diversos fatores como gratuidade, precisão dos dados do SRTM e pela grande cobertura disponível dos dados, uma vez que nem todas as regiões apresentam disponibilidade de dados cartográficos e que os resultados podem variar de acordo com a percepção humana.

Palavras-chaves: geoprocessamento, recursos hídricos, Shuttle Radar Topographic Mission (SRTM).

\section{ABSTRACT COMPARISON BETWEEN MANUAL AND AUTOMATIC DELIMITATION OF CORUPÁ STREAM WATERSHED, RS, BRASIL}

Several environmental impacts such as deforestation, soil erosion, water pollution and chaotic urban occupation are related to lack of planning in land use in watersheds. For proper planning and management of water resources is critical to proper delineation of watersheds. Thus, this study aimed to analyze a methodology for automatic delineation of watersheds in the basin of the creek Corupá, Rio Grande do Sul/RS, Brazil, using design data Shuttle Radar Topographic Mission (SRTM) and compare with the values obtained in the area of manual scanning with based on topographic maps with scale of 1:50 000. For this, the data were integrated and processed using the software ArcGIS 9.2 (ESRI, 2006). The methodology used in this process was divided into six 
Rev. Elet. em Gestão, Educação e Tecnologia Ambiental (e-ISSN: 2236-1170)

stages, as follows: filling depressions ("fill sinks"), flow direction ("flow direction"), accumulated flow ("flow accumulation"), extraction of drainage ("conditional - > con "), linking crossing stream ("stream link ") and delineation of watersheds ("watershed"). The basin area delimited from the contours of the topographical was $29.162,4$ ha and for the automatic delineation met total area of $28.472,2 \mathrm{ha}$, representing a difference of $2,4 \%$ of total area $690,2 \mathrm{ha}$. The results show very close values confirming be advantageous to automate the delineation of watersheds by several factors such as gratuity, the SRTM data accuracy and the great coverage of available data, since not all regions are data availability and the cartographic results may vary according to the human perception.

Keywords: geoprocessing, hydric resources, Shuttle Radar Topographic Mission (SRTM).

\section{INTRODUÇÃO}

A escassez de água potável no planeta deve-se principalmente a intensa urbanização ocorrida nas últimas décadas e o consumo deste bem em ritmo acelerado. Quadrado e Vergara (2003) apontam que existem duas ideologias sobre quem deveria gerenciar as reservas de água: de um lado as empresas com o poder de capital e de outro, os ambientalistas para quem a água não tem preço nem dono, pois pertence a todos. No entanto, a educação da população é tão importante quanto qualquer intervenção que governos ou empresas podem fazer. De acordo com a Organização das Nações Unidas (ONU, 2012), estima-se que um bilhão de pessoas no mundo carece de acesso a um abastecimento de água suficiente, definido como uma fonte que possa fornecer 20 litros por pessoa por dia a uma distância não superior a mil metros.

Uma das ações necessárias para evitar a escassez de água é promover uma melhor administração dos recursos hídricos em nível de bacias hidrográficas, utilizando para isso, tecnologias avançadas de monitoramento e gestão. Técnicas de sensoriamento remoto e sistemas de informações geográficas têm contribuído para o monitoramento dos recursos garantindo respostas rápidas e confiáveis.

Diante disso, este trabalho teve como objetivo analisar uma metodologia de delimitação automática de bacias hidrográficas na bacia do Arroio Corupá, RS, Brasil, utilizando dados do projeto Shuttle Radar Topographic Mission (SRTM) e comparar com os valores de área obtidos na digitalização manual com base em cartas topográficas, com escala de 1:50 000.

\section{METODOLOGIA}

A bacia do Arroio Corupá, encontra-se na região central do Rio Grande do Sul e compreende os municípios de Agudo, Sobradinho, Ibarama, Lagoa Bonita do Sul, Cerro Branco e Paraíso do sul. As coordenadas geográficas que nos dão referência de sua localização são 2932'58'S e 5313'50”W de Greenwich. A localização da bacia está representada na Figura 1. 
Rev. Elet. em Gestão, Educação e Tecnologia Ambiental (e-ISSN: 2236-1170)

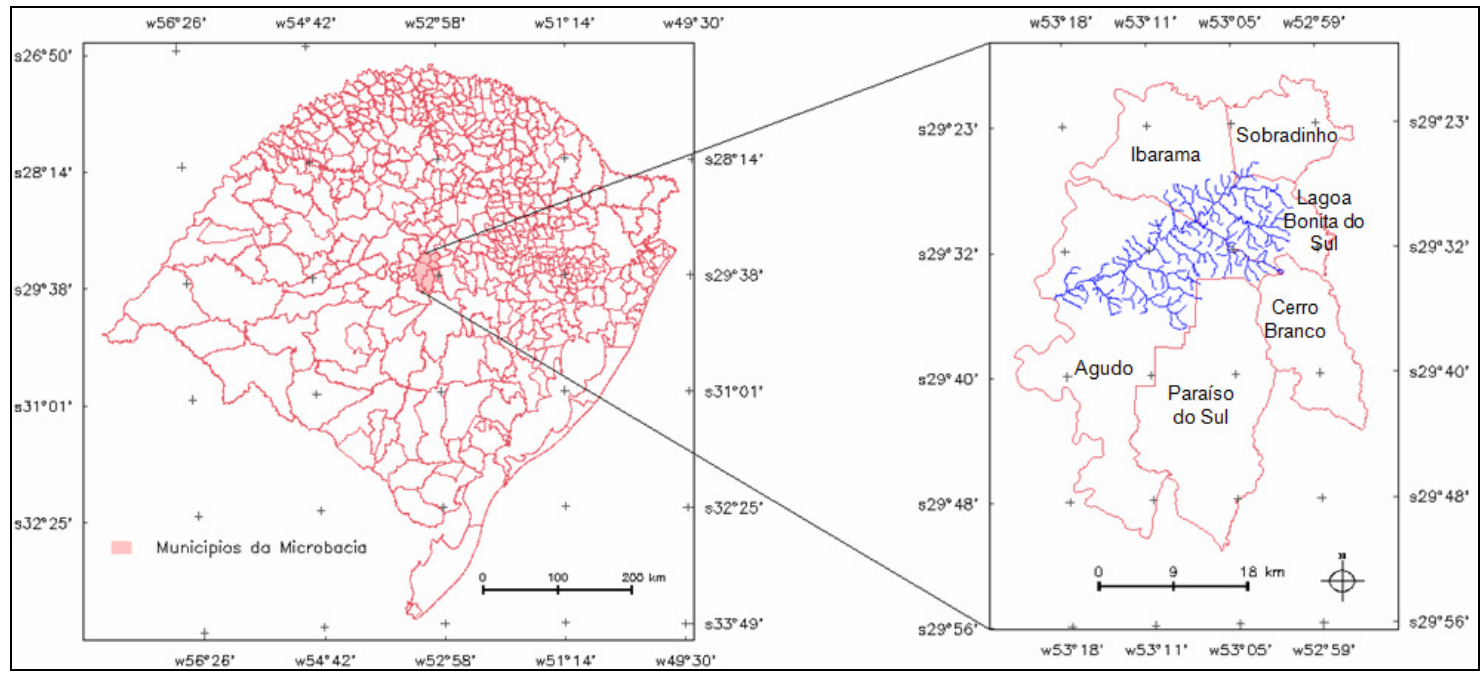

Figura 1. Localização da bacia do Arroio Corupá no estado do Rio Grande do Sul.

O Arroio Corupá é um afluente do rio Jacuí. A bacia hidrográfica do rio Jacuí tem área de $71.600 \mathrm{~km}^{2}$, que corresponde a 83,5 \% da área da região hidrográfica do Guaíba (FEPAM, 2010). 0 clima, segundo a classificação de Köppen, é Subtropical Temperado Úmido, do tipo "Cfa", caracterizado por possuir verões quentes com temperatura média de $22^{\circ} \mathrm{C}$, com precipitação em todos os meses do ano (MORENO, 1961).

Para o desenvolvimento desta pesquisa, foram utilizadas cartas topográficas com escala de 1:50 000, conforme apresentado na Figura 2, e dados SRTM disponíveis no site da Empresa Brasileira de Pesquisa Agropecuária (EMBRAPA, 2012). Para a área de estudo foi necessária uma carta: Agudo - SH.22-V-CV-2, MI-2966/2 (BRASIL,1980) conforme está representado na Figura 3. Para o tratamento dos dados foi utilizado o software ArcGIS 9.2 (ESRI, 2006).

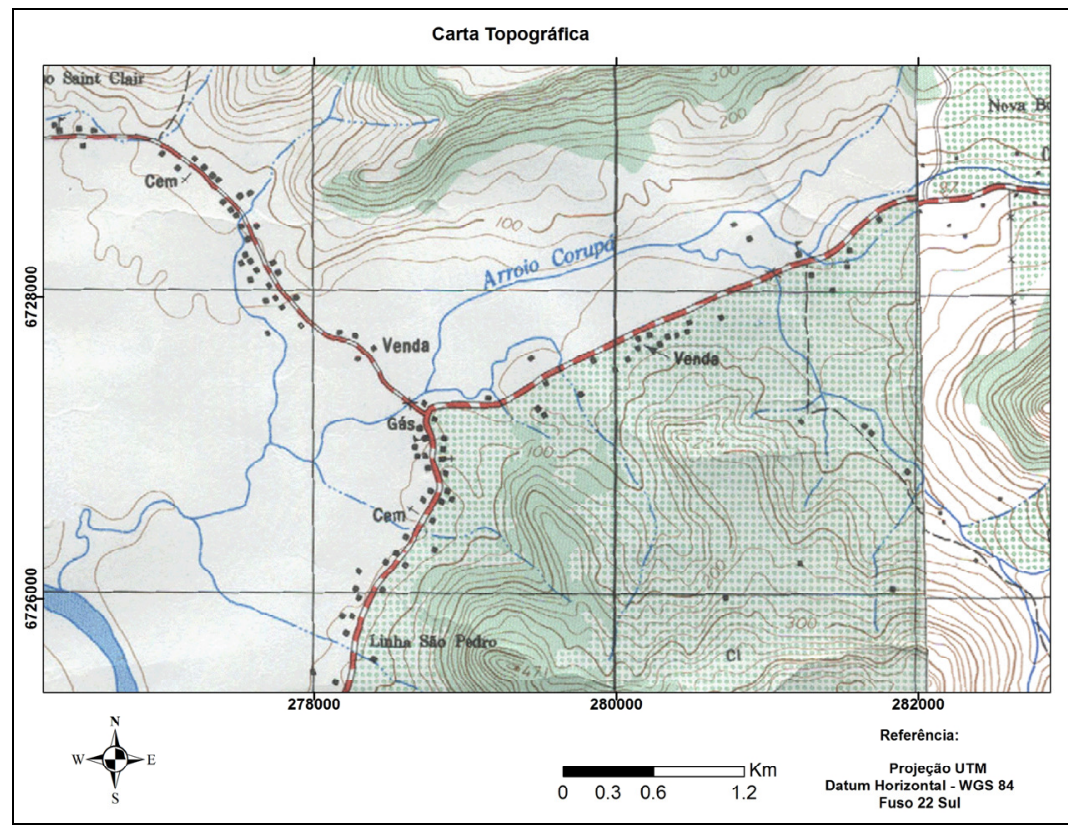

Figura 2. Carta topográfica de Agudo (SH.22-V-CV-2, MI-2966/2) utilizada para delimitação da drenagem e do limite da bacia a partir das curvas de nível. 


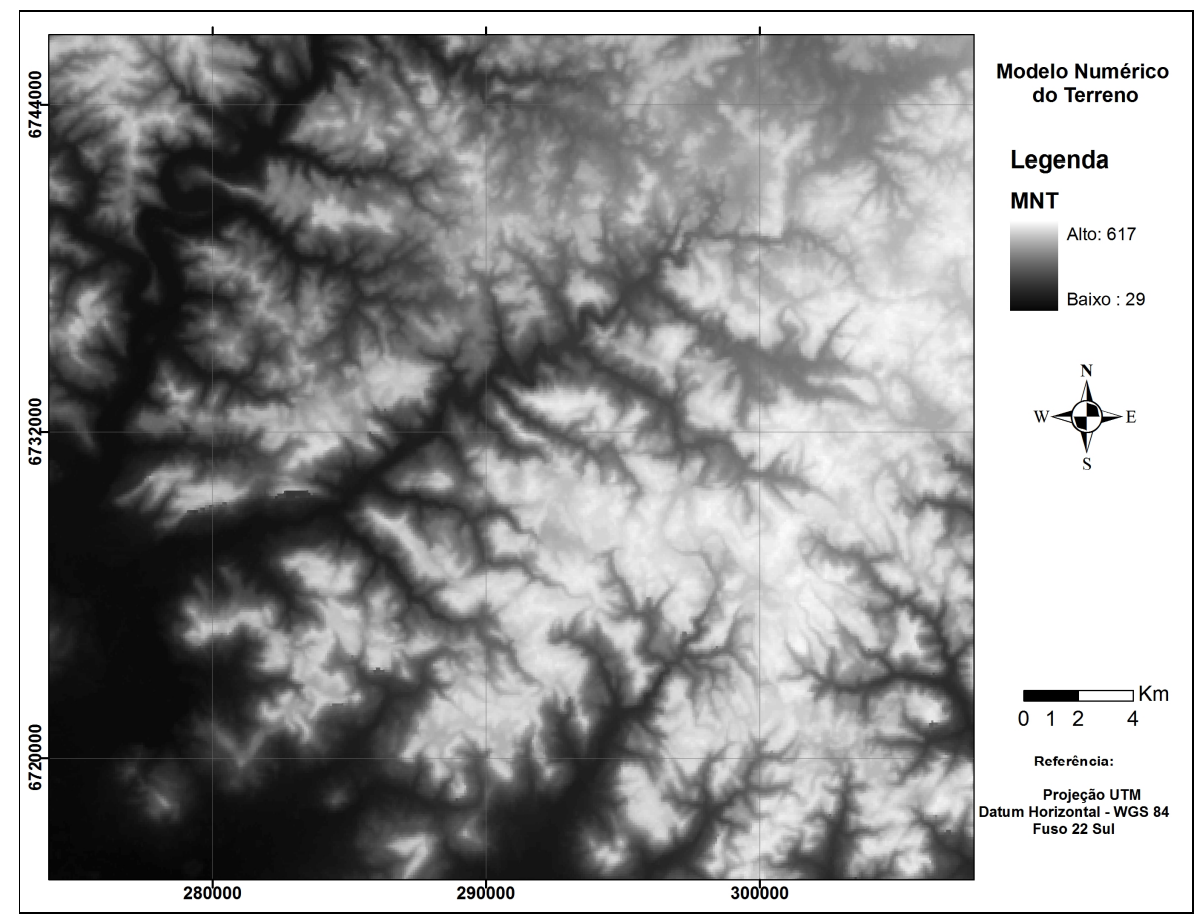

Figura 3. Mapa do MNT obtido a partir de dados SRTM.

O processo de delimitação manual da bacia hidrográfica foi desenvolvido utilizando as curvas de nível da carta topográfica. Tendo em vista a topografia do terreno é responsável pela drenagem da água da chuva e os limites entre as bacias hidrográficas encontram-se nas partes mais altas do relevo delimitou-se a bacia seguindo as curvas de nível de maior cota, simulando o trajeto das águas.

Para a realização da delimitação automática utilizou-se o software de geoprocessamento ArcGIS 9.2 e subdividiu-se em seis etapas, sendo: preenchimento de depressões ("fill sinks"), direção de fluxo ("flow direction"), fluxo acumulado ("flow accumulation"), extração de drenagens ("conditional -> con"), ligação de cruzamentos de fluxo ("stream link") e delimitação de bacias ("watershed").

Para a extração das redes de drenagem foi necessário primeiro converter as imagens SRTM, em formato raster, para o formato GRID. O próximo passo foi eliminar todas as depressões indevidamente geradas ao converter o MDE para GRID. O preenchimento dessas depressões é feito através do comando "fill" no ArcGIS 9.2. De acordo com Vieira et al. (2005), estas depressões impedem ou desviam o escoamento superficial o que acarretaria em uma delimitação errônea das bacias de contribuição.

A etapa seguinte é a geração de um mapa com a direção de fluxo da drenagem (Figura 4), através do comando "flow direction". De acordo com Guimarães (2010), a etapa de geração do arquivo de direção de fluxo é uma das etapas mais importantes para a extração da rede de drenagem e delimitação da bacia hidrográfica, uma vez que é onde são realizados os cálculos dás áreas de maior declividade, por onde o fluxo de drenagem é direcionado no meio ambiente, naturalmente. É importante lembrar que a água flui naturalmente pelo caminho de menor esforço, sendo assim quanto maior a declividade existente, menor será o esforço "exercido" pela 
água e desta forma serão estes valores de célula na imagem gerada que serão selecionados como caminhos das redes drenagem.

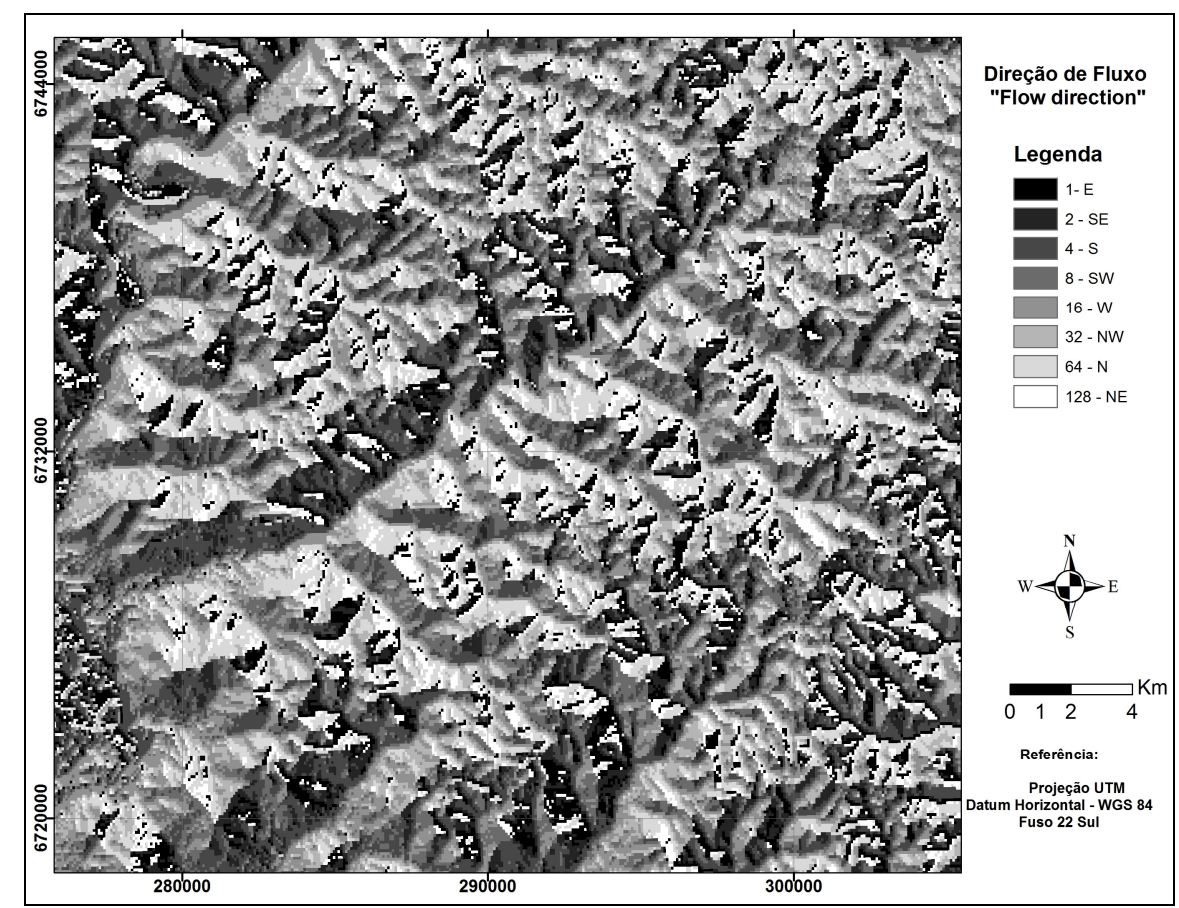

Figura 4. Mapa de direção de fluxo superficial.

A partir da geração do raster de direção de fluxo pode-se gerar o raster do fluxo acumulado (Figura 5). Segundo Valeriano (2008), o fluxo acumulado é um parâmetro que indica o grau de confluência do escoamento e pode ser associado ao fator comprimento de rampa aplicado em duas dimensões. 
Rev. Elet. em Gestão, Educação e Tecnologia Ambiental (e-ISSN: 2236-1170)

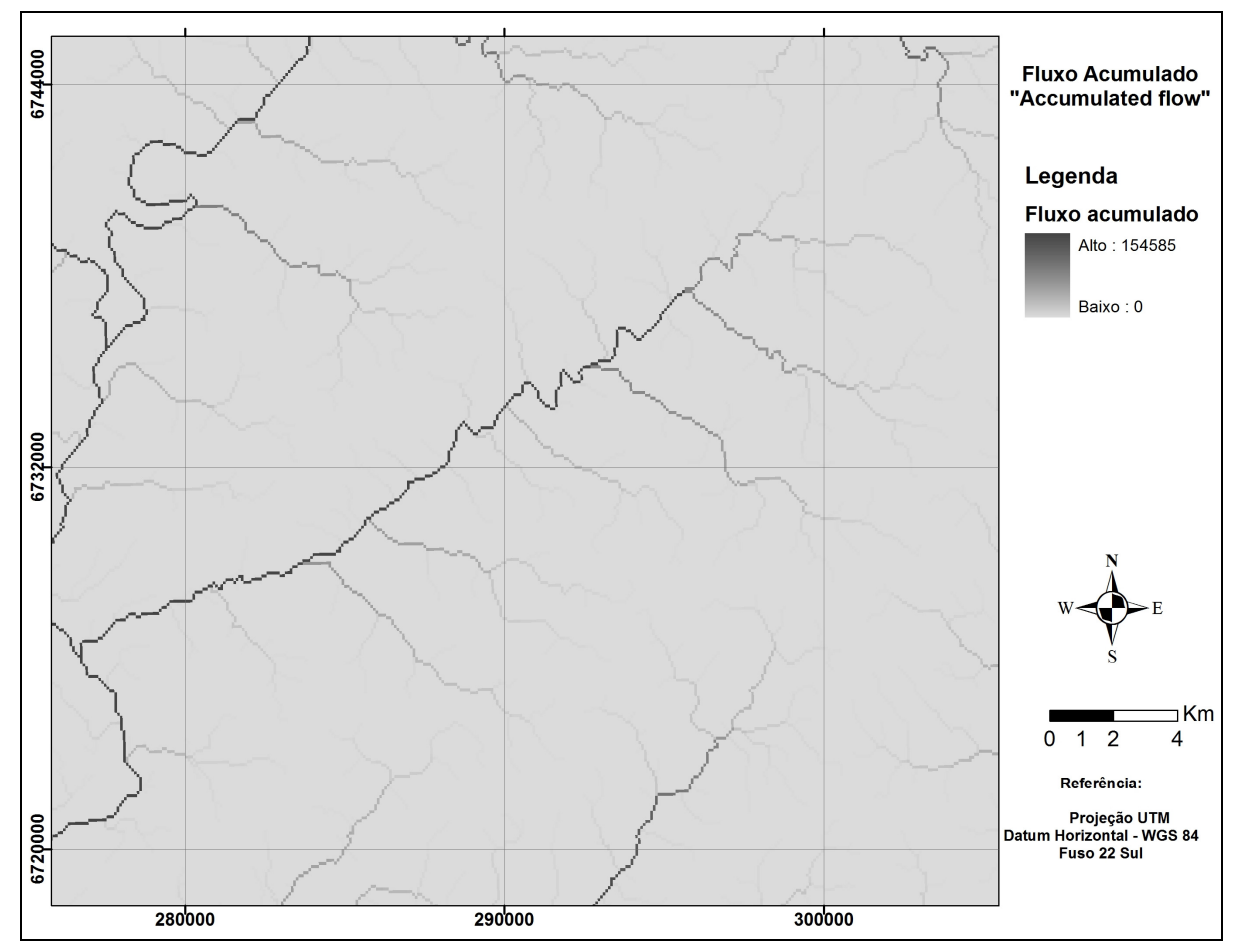

Figura 5. Mapa de fluxo superficial acumulado.

Após a geração dos raster de fluxo acumulado, gerou-se um arquivo representativo da rede de drenagem, por meio do comando "con", que limita a hidrografia às drenagens de maior acumulação de água. Nesse caso, utilizou-se o valor de fluxo acumulado maior que o limiar 100. De forma prática, quanto menor o limiar, menor será o tamanho das bacias hidrográficas a serem determinadas.

Através do comando "stream link" adicionou-se valores a cada trecho de drenagem gerado na etapa anterior, correspondentes a direção de fluxo da bacia. Em seguida, com o comando "watershed" delimitou-se as bacias automaticamente.

A última etapa foi a vetorização manual do limite da bacia do Arroio Corupá sobre o arquivo que continha as bacias delimitadas e por fim, a comparação entre esse limite gerado por meio dos dados SRTM e o limite obtido pela delimitação manual, levando em conta as curvas de nível de uma carta topográfica.

\section{RESULTADOS E DISCUSSÃO}

O limite da área de drenagem extraída a partir da delimitação manual sobre a carta topográfica e automática sobre os dados do SRTM está representado na Figura 6. 


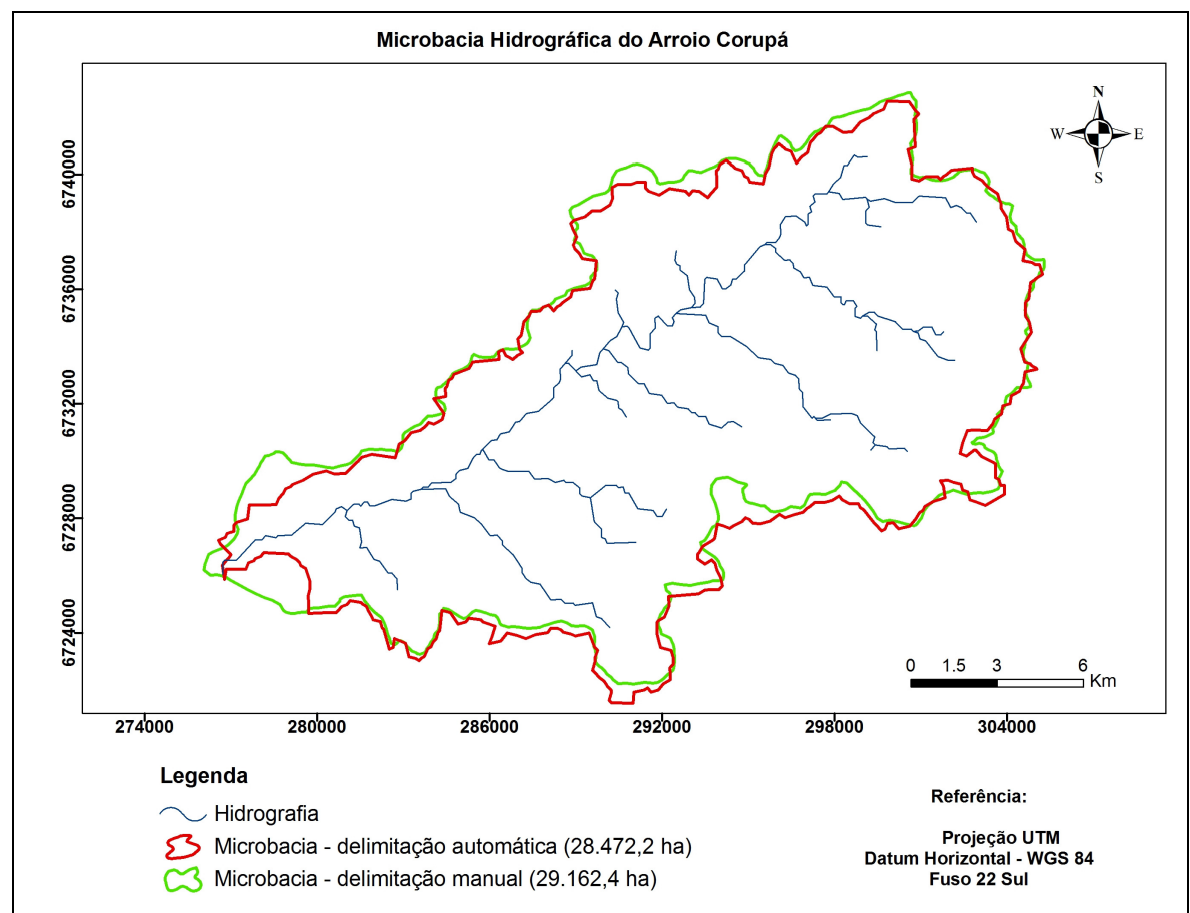

Figura 6. Bacia hidrográfica do Arroio Corupá demilitada de forma automática (a partir dos dados SRTM) e manual (a partir da orientação das curvas de nível).

A bacia hidrográfica do Arroio Corupá, resultante da delimitação automática e da delimitação manual constitui, respectivamente, área de $28.472,2$ ha e perímetro de $109,5 \mathrm{~km}$ e área de $29.162,4$ ha e perímetro de $102,9 \mathrm{~km}$. Comparando-se com os resultados obtidos em relação à delimitação manual a partir de curvas de nível da carta topográfica na escala de 1:50 000, verifica-se diferença, relativamente pequena, de 690,2 ha, o que dá em torno de 2,4\% de variação(Tabela 1).

Tabela 1. Áreas delimitadas na bacia do Arroio Corupá.

\begin{tabular}{cccc}
\hline Base de Dados & Metodologia & Área (ha) & Perímetro (km) \\
\hline $\begin{array}{c}\text { Carta topográfica } \\
\text { (Escala 1: 50 000) }\end{array}$ & Manual & $29.162,4$ & 102,9 \\
Dados SRTM & Automática & $28.472,2$ & 109,5 \\
\hline & Diferença & $\mathbf{6 9 0 , 2}(\mathbf{2 , 4 \% )}$ & $\mathbf{6 , 6 ( 6 , 0 \% )}$ \\
\hline
\end{tabular}

\section{CONCLUSÕES}

Dada a importância que as bacias hidrográficas vem ganhando no cenário ambiental, onde são consideradas como unidades de gestão territorial, a busca por alternativas viáveis na delimitação das mesmas é extremamente importante. Nesse sentido os resultados mostram que a delimitação automática da bacia do Arroio Corupá, no software ArcGIS 9.2, com base nos dados do SRTM, apresentou-se como metodologia perfeitamente aplicável. Em comparação com uma 
metodologia tradicionalmente usada na cartografia digital que é a delimitação de bacias através das curvas de nível de uma carta topográfica escaneada, os valores das áreas das bacias apresentaram uma diferença em torno de $2,4 \%$ e para o perímetro $6,0 \%$. O que pode ser considerado aceitável levando em conta que ambos os métodos possuem erros sistemáticos. Além disso, os dados do SRTM proporcionaram economia de tempo, através da praticidade da delimitação automática. Também é preciso ressaltar que esses dados são essenciais em regiões que não possuem registros cartográficos, compatíveis com as diferentes escalas de trabalho. Dessa forma, conclui-se que a metodologia proposta nesse trabalho mostrou-se bastante rápida e confiável.

\section{REFERÊNCIAS BIBLIOGRÁFICAS}

BRASIL. Ministério do Exército. Diretoria de Serviços Geográficos (DSG). Agudo. Carta Topográfica, Porto Alegre, 1980. F.SH-22-V-C-1/MI:2966/1. Escala 1:50000.

Empresa Brasileira de Pesquisa Agropecuária (EMBRAPA). Monitoramento por satélite. Disponível em: http://www.relevobr.cnpm.embrapa.br/download/index.htm. Acesso em: 14 jul. 2012.

ESRI. ENVIRONMENTAL SYSTEMS RESEARCH INSTITUTE, ArcGIS Professional GIS for the desktop, version 9.2. Software. 2006.

Fundação Estadual de Proteção Ambiental Henrique Luiz Roessler -RS (FEPAM). Qualidade das águas da bacia hidrográfica do Rio Jacuí. Disponível em:

<http://www.fepam.rs.gov.br/qualidade/qualidade_jacui/jacui.asp>. Acesso em: 15 jul. 2012.

GUIMARÃES, A. A. Gestão dos recursos Hídricos. .Net. Disponível em: $<$ http://blog.geoprocessamento.net/2010/07/delimitacao-de-bacias-hidrograficas-e-a-extracaode-redes-de-drenagem/>. Acesso em: 14 set. 2012.

MORENO, J.A. Clima do Rio Grande do Sul. Porto Alegre: Secretaria da Agricultura, 1961. 73p.

ONU (Organização das Nações Unidas). A ONU e a água. Disponível em: http://www.onu.org.br/a-onu-em-acao/a-onu-em-acao/a-onu-e-a-agua/. Acesso em: 25 jul.2012.

QUADRADO, A.; VERGARA, R. Vai Faltar Água?. Super Interesssante. Rio de Janeiro, ed. Abril, $\mathrm{n}$ 189, p.42-46, 2003. Disponível em:

http://super.abril.com.br/saude/vai-faltar-agua-443923.shtml. Acesso em: 11 dec.2012.

VALERIANO, M.M. Dados topográficos. In: FLORENZANO, T.G. (Org.). Geomorfologia, conceitos e tecnologias atuais. São Paulo: Oficina de Textos, 2008. p.72-104.

VIEIRA, E. M.; ALMEIDA, F. T.; ALVES, M. G.; Uso do MDT e Bacias de contribuição (Watershed) na seleção de pontos para o monitoramento da contaminação agrícola; In: SIMPÓSIO BRASILEIRO DE SENSORIAMENTO REMOTO, 12, 2005, Goiânia. Anais... São José dos Campos: INPE, 2005. Artigo, p. 3427-3432. 